\title{
LIMITACIONES Y DIFICULTADES DE LA PANDEMIA COVID-19 EN LA EDUCACIÓN UNIVERSITARIA
}

\author{
María de Lourdes Vázquez Arango \\ Profesor-Investigador \\ Universidad Autónoma Benito Juárez de Oaxaca \\ https://orcid.org/0000-0001-6249-9677 \\ mar.v.aa@hotmail.com \\ Ricardo Alarcón Alcántara \\ Profesor-Investigador. \\ Universidad Autónoma Benito Juárez de Oaxaca. \\ https://orcid.org/0000-0001-6584-7657 \\ rialal2@hotmail.com \\ Eric Amín Ramírez Castillo \\ Profesor-Investigador \\ Universidad Autónoma Benito Juárez de Oaxaca \\ https://orcid.org/0000-0002-8762-1509 \\ aminramirez@gmail.com
}

\author{
Para citar este artículo puede utilizar el siguiente formato: \\ María de Lourdes Vázquez Arango, Ricardo Alarcón Alcántara y Eric Amín Ramírez Castillo: \\ "Limitaciones y dificultades de la pandemia Covid-19 en la educación universitaria", Revista Caribeña de \\ Ciencias Sociales (vol 10, № 7 julio-septiembre 2021, pp. 208-223. En línea: \\ https://doi.org/10.51896/caribe/RFVR3475
}

\section{RESUMEN}

Es importante analizar la situación prevaleciente en las instituciones educativas durante la contingencia sanitaria originada por la pandemia de COVID-19. Considerando la gravedad de la situación, las autoridades federales, estatales y municipales, se vieron obligadas a suspender las clases presenciales, teniendo que migrar a clases a distancia a través de medios virtuales. El estudio se realiza en los alumnos de la Facultad de Contaduría y Administración de la Universidad Autónoma Benito Juárez de Oaxaca. El objetivo principal radica en identificar las limitaciones y dificultades de la pandemia en los alumnos a nivel universitario durante la emergencia sanitaria COVID-19. Se plantea como pregunta de investigación: ¿Qué limitaciones y dificultades enfrentan los alumnos universitarios de la Facultad de Contaduría y Administración en época de pandemia? Se parte del supuesto si al emigrar a las clases virtuales los alumnos consideran que no obtendrá el mismo conocimiento y su aprendizaje se verá disminuido. El estudio es de carácter exploratorio, busca comprender información relacionada con el 
fenómeno registrando y analizando las principales limitaciones y dificultades que presentan los alumnos universitarios en situación de pandemia, apoyando en datos estadisticos recopilados de entrevistas y estadisticas de la Facultad de Contaduría y Administración.

Palabras clave: Educación a distancia, Educación Profesional, Pandemia, Tecnologías de la Información, Tecnología educativa.

\section{LIMITATIONS AND DIFFICULTIES OF THE COVID-19 PANDEMIC IN UNIVERSITY EDUCATION}

\section{ABSTRACT}

It is important to analyze the prevailing situation in educational institutions during the health contingency caused by the COVID-19 pandemic. Considering the seriousness of the situation, the federal, state and municipal authorities were forced to suspend face-to-face classes, having to migrate to distance classes through virtual means. The study is carried out in the students of the Faculty of Accounting and Administration of the Autonomous University Benito Juárez de Oaxaca. The main objective is to identify the limitations and difficulties of the pandemic in students at the university level during the COVID-19 health emergency. It is posed as a research question: What limitations and difficulties do university students of the Faculty of Accounting and Administration face in times of a pandemic? It is assumed that when students emigrate to virtual classes, they consider that they will not obtain the same knowledge and their learning will be diminished. The study is exploratory in nature, seeks to understand information related to the phenomenon by recording and analyzing the main limitations and difficulties faced by university students in a pandemic situation, based on statistical data collected from interviews and statistics from the Faculty of Accounting and Administration.

Key words: Distance Education, Professional Education, Pandemic, Information Technology, Educational Technology

JEL: D8, D83, I0, 11,12,I3

\section{INTRODUCCIÓN}

La Comisión Económica para América Latina y el Caribe [CEPAL] (2020), ha señalado que previo a esta situación de pandemia, los índices de pobreza y marginación tenían una tendencia en aumento, demostrando la creciente desigualdad. Por su parte, la Organización de las Naciones Unidas [ONU] (2020) ha señalado que a raíz de la pandemia los abismos sociales, económicos y tecnológicos 
se han hecho más evidentes, específicamente en el sector educativo donde las afectaciones repercuten en los estudiantes, docentes, personal directivo y administrativo. ${ }^{1}$

Investigaciones realizadas por el Consejo Nacional de Evaluación de la Política de Desarrollo Socia [CONEVAL](2015) señalan que cerca del $54.2 \%$ de la población oaxaqueña presenta una o más carencias sociales, representando una población a nivel Estado de 806,988 personas; mientras que un $14 \%$ son personas vulnerables por ingreso al ser igual o inferior a la línea de bienestar. Estos datos también muestran repercusiones en el aspecto educativo existiendo un rezago en la población que no cuenta con educación básica obligatoria completa (primaria o secundaria) y que en gran medida resta la oportunidad de avanzar en los niveles educativos subsecuentes.

Sin lugar a duda, la educación además de ser un derecho inalienable y una posibilidad de acceso para todos es un bien común para impulsar sociedades justas, inclusivas e igualitarias y no ha sido ajena a los efectos colaterales de esta emergencia sanitaria. Partiendo de esta problemática, es posible señalar que las autoridades en sus diversos niveles han adoptado diversas estrategias ante el COVID-19, señalando principalmente: 1) Suspensión de clases presenciales; 2) Despliegue de modalidades enseñanza-aprendizaje a distancia, apoyadas en la mayor parte de tecnologías, formatos y plataforma; 3) Apoyo y movilización del personal a sus comunidades de origen o carentes de tecnología; y 4) Atención a la salud y bienestar de estudiantes y profesores (CEPAL-UNESCO, 2020).

Es una realidad que, en todos los niveles educativos no existía la preparación para una situación de esta magnitud, la falta de previsión y visión para apoyar tecnológicamente a los planteles educativos, así como procesos de capacitación adecuados para manejo de plataformas sincrónicas y asincrónicas han forzado a improvisar la educación en esta modalidad. En México a diferencia de otros países que estaban al inicio del ciclo escolar, decretar la suspensión de actividades presenciales a mitad del ciclo, no permitió retomar un ritmo adecuado y estrategias ideales para su desarrollo.

Sin embargo, las suspensión de actividades relacionadas con la academia no solo tienen repercusiones en el proceso de enseñanza-aprendizaje (CEPAL, 2020), también involucra la disminución del flujo económico de las zonas cercanas a las instituciones educativas, disminución de capacidad económica por parte de las familias, aumento de riesgo de violencia contra integrantes de la familia en especial del sexo femenino e infantes, sobrecarga de trabajo, entre otras cuestiones que afectan en el aspecto personal, psicológico y emocional (CEPAL-UNESCO, 2020).

\footnotetext{
${ }^{1}$ En América Latina y el Caribe a nivel superior nivel superior se registran afectaciones para 23.4 millones de estudiantes y 1.4 millones de docentes (Instituto Internacional para la Educación Superior en America Latina y el Caribe [IESALC], 2020:12).
} 
La investigación se centra en la educación a nivel superior cuestionando ¿Qué limitaciones y dificultades se presentan en alumnos de educación universitaria en situación de pandemia? Sobre todo, al tomar en cuenta que la población preuniversitaria busca la continuidad de sus estudios fuera de una de las ocho regiones que integra el Estado (Gobierno del Estado de Oaxaca, 2020); al existir mayor oferta educativa, teniendo como primera opción la máxima casa de estudios a nivel estatal y este suceso acontecido por el coronavirus SARS-Cov 2 será catalizador para una educación resiliente capaz de afrontar cualquier tipo de situación y garantizar pese a los riesgos que se presenten coordinación, liderazgo y comunicación entre sus actores. (Organización de las Naciones Unidas [ONU], 2020:4).

\section{Los efectos de la pandemia en la Educación Universitaria}

La disrupción que ha provocado la pandemia de COVID-19 en todos los sectores, ha afectado el orbe, especialmente el sector educativo se ha forzado a cerrar aulas y con ello contener esa extensa familia de virus, en particular el coronavirus identificado en la ciudad china de Wuhan, denominado SARS- CoV-2 o COVID-19, que tiene gran capacidad de contagio, resistencia y mutación. (Organización Panamericana de la Salud, 2020).

CEPAL y UNESCO (2020), han puesto especial interés en monitorear el impacto del Coronavirus en la educación, señalan las graves disparidades educativas preexistentes y ahora la reducción de oportunidades a sectores vulnerables como zonas pobres o rurales. Se han manifestado diversos obstáculos como baja conectividad, falta de contenido alienado y falta de preparación de los catedráticos ante esta nueva realidad. (ONU, 2020). La CEPAL y UNESCO (2020), llegan incluso a señalar que los avances llevados a cabo en las últimas décadas afectarán a las generaciones actuales y futuras, generando rezagos sustanciales en los procesos aprendizaje del alumnado.

Sin embargo, no solo es analizar aspectos negativos, algo bueno que ha traído consigo esta situación es la capacidad de innovación que ha demostrado el sector educativo. Se ha recurrido a la radio, televisión, redes sociales, y material de apoyo para dar respuesta a la ausencia de actividades presenciales; así mismo se ha creado una red de apoyo a docentes para poder hacer frente a la carencia de conocimiento tecnológico por medio de diversos canales y plataformas dando sugerencias y apoyo por parte de otros integrantes de la comunidad e incluso actores gubernamentales para poder resanar en la medida de lo posible esas carencias mostradas (ONU, 2020).

Pese a ello, a nivel universitario, los bajos niveles de digitalización, deficiencias estructurales tecnológicas, infraestructura tecnológica y alfabetización digital alumno-profesor (ONU, 2020) dificultan el proceso, tomando en cuenta que existen carreras que deben llevar a cabo prácticas y formarse profesionalmente con actividades que difícilmente pueden ser sustituidas por videos o clases en línea. A 
ello hay que sumar a estudiantes en condiciones de vulnerabilidad que difícilmente tienen acceso a conectividad y a equipos de computo. Haeck y Lefebvre (2020), consideran que la brecha en relación con las competencias socieconomicas puede verse incrementada en un $30 \%$ posterior a la pandemia, por lo que las afectaciones en el proceso de aprendizaje puede verse gravemente afectada.

En ese sentido, el Banco Mundial (2020), coincide que el cierre de escuelas trae consigo en el presente y futuro pérdidas y reducciones significativas en materia de aprendizaje; incremento de la desigualdad en poblaciones ya de por sí marginadas, incremento de la desersion escolar y baja capacidad de alcance de competencias necesarias para participación eficaz, productiva y de aprendizaje en la sociedad. Esta interrupción también afecta los servicios sanitarios y psicosociales al ser las instituciones educativas plataformas de canalización, prevención, diagnóstico y asesoría para los jóvenes universitarios (ONU, 2020).

\section{El papel del docente ante la pandemia}

En el transcurso de la crisis provocada por la pandemia, la exigencia hacia los docentes de tomar las medidas necesarias para continuar con el proceso de enseñanza se hizo presente. Se requirió la puesta en marcha de mecanismo que pudieran hacer frente a la situación, sin que previamente existiera una preparación, capacitación o los recursos materiales y tecnológicos mínimos necesarios para hacer frente a la contingencia.

Los docentes, tuvieron que enfrentarse a una situación que requería innovación y capacidad de poder enfrentar los procesos de comunicación necesarios con sus alumnos, incluida la mensajería o correos electrónicos en primer instancia. Se hizo evidente la falta de competencias digitales básicas por parte de muchos maestros, demostrando que incluso con infraestructura y conectividad adecuadas las aptitudes básicas en TIC dificultaría la educación a distancia. (ONU, 2020).

Se hicieron evidententes las condiciones laborales de muchos docentes, en el caso de instituciones privadas se mostró la falta de contratos, salarios dignos versus la exigencia en las cargas de trabajo para evitar la salida de los alumnos y espacios adecuados para el desempeño de su labor. Si las escuelas particulares llegaran a cerrar por su falta de capacidad económica; serán las instituciones federales y estatales las que tendrán que absorber a los estudiantes adicionales si su capacidad, infraestructura y recursos económicos lo llegan a permitir, de lo contrario este gran número de alumnos deberán suspender su preparación académica.

En el caso de los docentes que no tienen garantizado un contrato, deberán buscar otras fuentes de empleo, lo que reducirá la escasez de personal capacitado para poder otorgar servicios educativos de 
calidad. Ahí se demuestra la importancia para invertir en programas de capacitación y garantizar condiciones dignas para los maestros en todos los niveles educativos (ONU, 2020).

Las condiciones de retorno a las aulas a más de un año suspensión de actividades se considera incierto. A inicios de mayo de 2021 se dio inicio al programa de vacunación para docentes y personal que labora en las insituciones de educación a nivel administrativo y directivo. Sin embargo lo referente a la base estudiantil y su entorno cercano aún esta pendiente. Hay que recordar que el virus sigue circulando y mutando agresiva y permanentemente, lo que anula la garantía de retornos seguros a las aulas.

En varios países se tiene previsto un modelo híbrido o mixto de enseñanza, reduciendo grupos y tomando clases al aire libre, aunado a las medidas de protección básicas necesarias para evitar contagios (ONU, 2020); sin embargo las condiciones en México y principalmente en Oaxaca, teniendo en cuenta sus caracteristicas institucionales, de sobrepoblación escolar y carencia de docentes, dificultarán poner en marcha modelos similares e incluso planear adecuadamente incremento en la contratación de personal docente, incremento en los horarios de clase, ampliación de periodos escolares, introducción de programas de recuperación y aprendizaje acelarado, así como la adaptación de planes de estudio de acuerdo al rezago presentado por los alumnos.

Consecuentemente es importante garantizar una mejor preparación para los docentes en aspectos pedagógicos, aprendizaje diferenciado y de evaluación significativa y equitativa, aunado a un programa consistente de alfabetización digital; para así garantizar una igualdad e inclusión en el proceso enseñanza-aprendizaje.

\section{Estrategias planteadas por la UABJO ante el COVID-19}

La política por COVID-19 en la Universidad Autónoma "Benito Juárez" de Oaxaca, fue comunicada el día 17 de marzo de 2020, a través de una reunión con autoridades de la Administración Central y el rector de la Universidad el Dr. Eduardo Bautista Martínez, para atender con responsabilidad y compromiso la nueva situación. Se instaló además la Comisión Técnica de Medidas de Salud (CTMS) cuya función es realizar acciones de prevención sobre el COVID-19, conforme lo dispuesto en el acuerdo 02/03/20 publicado en el Diario Oficial de la Federación. Otra de las medidas fue la reorganización de actividades académicas para realizar ajustes al calendario escolar y el trabajo a distancia por medio de plataformas digitales (Universidad Autónoma Benito Juárez de Oaxaca, 2020), así como la creación Sistema Institucional de Actualización de Datos (SIAD) como medio de comunicación entre personal docente, estudiantil y administrativo con la Administración Central.

Se realizó la actualización de equipos de video vigilancia para salvaguarda la seguridad institucional, mientras que la Dirección de Redes, Telecomunicaciones e Informática (DRTI) habilitó y 
puso a disposición de docentes y estudiantes el sitio "Herramientas Virtuales" para el aprendizaje en línea, la cual contempla: Aulas Colaborativas, Microsoft Teams, Google Clasroom, grupos privados de Facebook, Quizizz, Zoom, Webex Meetings, Encuesta Fácil y GoConqr, para dar seguimiento a los contenidos académicos en los planes y programas de estudio de nivel medio superior y superior. De igual manera con apoyo de Radio Universidad se dio impulso y difusión a diversos programas, campañas y cápsulas radiofónicas sobre temas relacionados con salud y educación (Universidad Autónoma Benito Juárez de Oaxaca, 2020).

También se da a conocer el sitio web www.recursosdigitales.anuies.mx de la Asociación Nacional de Universidades e Instituciones de Educación Superior (ANUIES), que cuenta con recursos académicos digitales, plataformas para reuniones y aulas virtuales, así como espacios de trabajo en la nube. Por otra parte, en apoyo a la comunidad docente se dan cursos en línea como: Modelo Educativo UABJO para la transformación social, a fin de facilitar la transversalización curricular, así como otros cursos para el aprendizaje de la comunidad universitaria docentes y estudiantes para orientaciones pedagógicas en tiempo de contingencia sanitaria por COVID-19 (Universidad Autónoma Benito Juárez de Oaxaca, 2020).

Preocupada por el bienestar de la comunidad universitaria, la administración central puso a disposición de docentes, alumnos y personal administrativo líneas telefónicas de apoyo psicológico, orientación y atención en situaciones de violencia, asesoría, atención y acompañamiento en posible violación a los derechos en contexto de pandemia y bibliotecas virtuales. Se implementó un canal souncloud para dar a conocer entrevistas con especialistas y también se abre un canal de videos "ejercítate en casa" para apoyar a la comunidad en general a la activación física y alimentación equilibrada (Universidad Autónoma Benito Juárez de Oaxaca, 2020 b).

Referente a la Facultad de Contaduría y Administración, apegados a los lineamientos nacionales, estatales y por parte de la Rectoría de la Universidad, se implementó el apoyo de plataformas digitales para dar seguimiento a los contenidos académicos. Al inicio del semestre se aplicó una encuesta para identificar los aprendizajes en el uso técnico y pedagógico de tecnologías con fines educativos, y planear cursos y talleres para el fortalecimiento de estos temas. Se acordó que la nueva modalidad para exámenes profesionales de licenciatura, maestría y doctorado se realizarían a través de plataformas tecnológicas. Para aquellos estudiantes sin acceso a internet o equipos de cómputo, se implementaron otro tipo de estrategias tanto para el seguimiento en sus avances académicos como los esquemas de evaluación (UABJO, 2020 b).

\section{METODOLOGÍA}


El trabajo de investigación es de carácter exploratorio, a fin de poder estudiar un problema que no es definido en su totalidad al ser una situación que no se había vivido previamente, por lo que puede considerarse que no existen resultados concluyentes. Se recogió información primaria a través de encuestas en google forms a los alumnos de la Facultad de Contaduría y Administración en la Universidad Autónoma Benito Juárez de Oaxaca, sobre los efectos que tuvo en ellos y en su formación académica la pandemia del COVID-19. La investigación secundaria se basó en la recopilación de información publicada sobre COVID-19, educación universitaria, repercusiones en la Facultad de Contaduría y Administración y afectaciones de la pandemia.

Del total de la población objeto de estudio que comprende a 3,876 alumnos de la Facultad de Contaduría y Administración, divididos 1,571 en la Licenciatura en Administración; 1,953 en la Licenciatura en Contaduría Pública; 330 en la Licenciatura en Turismo y Desarrollo Sustentable; 3 en la Licenciatura de Negocios y Microfinanzas y 19 en la Licenciatura en Administración Pública y Gestión Municipal. Se aplicaron encuestas a quienes estaban cursando el segundo, cuarto, sexto y octavo semestre de las carreras.

Se realizó un muestreo no probabilístico por la complicación de movilidad existente, la recolección de información se obtuvo mediante la aplicación de 583 encuestas, siendo todas validas, lo que representa una cobertura del $15 \%$ de la población total; la encuesta constó de 16 preguntas, las respuestas de estos ítems permitieron ampliar el panorama de la situación académica actual de los alumnos de la Facultad de Contaduría y Administración de la Universidad Autónoma Benito Juárez de Oaxaca.

Para fines del estudio se consideraron las siguientes categorías de donde se desprenden los ítems de las encuestas y que permiten señalar los resultados más relevantes según: Distribución de alumnos por región geográfica; Características de la zona: rural, urbana; Tecnologías de Información y Comunicación empleadas por los alumnos; conectividad; transición y adaptabilidad de modalidad presencial a virtual; alfabetización digital alumno-profesor; compromiso alumno-profesor; limitaciones y dificultades.

\section{RESULTADOS Y DISCUSIÓN}

Las características de la población objeto de estudio en un mayor porcentaje corresponde a las licenciaturas en administración y contaduría. La muestra que representa el 15\% de la población permitió obtener resultados significativos, y que muestran un panorama general de las limitaciones a la que se enfrentan los estudiantes universitarios en tiempos de pandemia. La tabla $1 \mathrm{da}$ a conocer las licenciaturas que corresponden a la Facultad de Contaduría y Administración, así como el total de alumnos que integran cada una y la representación de la muestra de encuestas.

\section{Tabla 1}


Total, de Alumnos y muestras de la Facultad de Contaduría y Administración.

\begin{tabular}{lcccc}
\hline $\begin{array}{c}\text { Licenciaturas de la Facultad de Contaduría y } \\
\text { Administración (FCA) }\end{array}$ & $\begin{array}{c}\text { Alumnos por carrera } \\
\text { Total } \\
(\mathbf{3 , 8 7 6 )}\end{array}$ & $\begin{array}{c}\text { Representación } \\
\text { de muestra }\end{array}$ & $\begin{array}{c}\text { Total } \\
\mathbf{( 5 8 3 )}\end{array}$ & $\begin{array}{c}\text { Encuestas aplicadas } \\
\text { Porcentaje }\end{array}$ \\
\hline Administración & 1,571 & $16 \%$ & 244 & $42 \%$ \\
Contaduría & 1,953 & $13 \%$ & 251 & $43 \%$ \\
Turismo y Desarrollo Sustentable & 330 & $25 \%$ & 83 & $14 \%$ \\
Negocios y Microfinanzas & 3 & $33 \%$ & 1 & $0.4 \%$ \\
Administración y Gestión Municipal & 19 & $21 \%$ & 4 & $0.6 \%$ \\
\hline Nota: Se tiene un total de 3,876 alumnos en la Facultad de Contaduría y Administración, que son divididos en cinco carreras y \\
donde el mayor número corresponde a la licenciatura en Administración y Contaduría, de donde se extrajo de igual manera una \\
muestra mayor para la aplicación de las encuestas. Con base en informe de la Dirección de la FCA y encuestas aplicadas a los \\
alumnos de la FCA-UABJO.
\end{tabular}

Respecto al semestre cursado, los alumnos encuestados en un $30 \%$ pertenecían a sexto semestre, $27 \%$ al segundo semestre, $22 \%$ cuarto semestre, y el $21 \%$ de octavo semestre.

Un parámetro importante que evaluar corresponde a la zona geográfica de origen de los alumnos. Las condiciones económicas de los alumnos, obligó a la mayoría de ellos a regresar a sus comunidades para disminuir sus gastos de manutención. Oaxaca se compone de ocho regiones, divida en 30 distritos, dentro de los cuales se ubican 570 municipios. La tabla 2 muestra la región y alumnos encuestados que corresponden a cada una de ellas.

\section{Tabla 2}

Distribución de Alumnos por Región

\begin{tabular}{|c|c|c|c|}
\hline & Región & $\begin{array}{c}\text { Alumnos } \\
(583)\end{array}$ & Porcentaje \\
\hline 1 & Valles Centrales & 358 & $61 \%$ \\
\hline 2 & Costa & 72 & $12 \%$ \\
\hline 3 & Mixteca & 44 & $8 \%$ \\
\hline 4 & Sierra Sur & 43 & $7 \%$ \\
\hline 5 & Sierra Norte & 29 & $5 \%$ \\
\hline 6 & Istmo & 18 & $3 \%$ \\
\hline 7 & Cañada & 10 & $2 \%$ \\
\hline
\end{tabular}

Nota: Con base en datos arrojados por las encuestas, es posible observar que un mayor porcentaje de la población estudiantil pertenece a Valles Centrales en un $61 \%$, seguidos por el resto de las regiones que representa el $39 \%$ de los alumnos distribuidos en la Costa, Mixteca, Sierra Sur, Sierra Norte, Istmo y Cañada. Información recabada con las encuestas aplicadas a los alumnos de la FCA-UABJO.

Otro dato importante tiene que ver con las características de la zona en la que radican los alumnos, obteniendo información a través de las encuestas que señalan que 331 alumnos, es decir un $57 \%$ viven en zonas urbanas y 252 alumnos que representan un $43 \%$ en zonas rurales. Así también se encontró que el $57 \%$ de los alumnos regresaron a su comunidad de origen y el $42 \%$ reside en la ciudad de Oaxaca y solo el 1\% realiza viajes entre la ciudad de Oaxaca y su lugar de origen. 
Si se toma en cuenta que en Oaxaca la conexión a internet en zonas urbanas es inestable, pese a que la mayoría de los servicios se encuentran ubicados en esos centros, en las áreas rurales la problemática se magnifica y las dificultades de conexión se vuelve más marcadas.

Algo importante a señalar, tiene que ver con la transición y adaptabilidad en el cambio de modalidad presencial a una modalidad virtual, apoyada en las Tecnologías de Información y Comunicación, por medio de las encuestas se manifiesta que 91 alumnos que representan el 16\% señalan que los profesores asignados cuentan con la capacidad y saberes digitales necesarios para enfrentar esta modalidad. Por otro lado 492 alumnos que consiste en el 84\% señalan las carencias por parte de los catedráticos para la migración adecuada a este tipo de enseñanza, hechos que generan frustración: falta de atención y compromiso en el proceso enseñanza-aprendizaje.

En este sentido, se hizo importante conocer si el personal docente tuvo la capacidad de adaptar los contenidos de los programas presenciales a la modalidad virtual. Como se había señalado en el caso en particular de México, las medidas de suspensión de actividades académicas se realizaron a mitad de semestre; lo que permitió contrastar esa fase con el inicio del siguiente periodo escolar, y así poder tomar las estrategias pertinentes para mejora del proceso de enseñanza.

En la primera fase que correspondió al semestre 2020-1, se detectó que los catedráticos de la Facultad de Contaduría y Administración debían tomar cursos o prepararse en el uso de las tecnologías y así estar preparados para, en caso de ser necesario mejorar su técnica de enseñanza el próximo semestre 2020-2, ya que el $70 \%$ de los alumnos encuestados manifestó que los catedráticos no estaban preparados para cumplir sus contenidos; en contraparte se tiene que el $22 \%$ si estaba preparado para enfrentar esta situación ya que previamente habían trabajado con herramientas tecnológicas y actividades asincrónicas, 46 de ellos que representa el $8 \%$ señaló que, aunque existía poca adaptabilidad y compaginación entre ambas partes para poder adquirir el conocimiento necesario en cada una de sus materias.

De los 583 alumnos encuestados, 382 que representa el 66\% hacen mención que si tienen acceso a tecnologías de información y comunicación para poder llevar a cabo sus actividades académicas; en contraparte 201 alumnos que equivale a un $34 \%$ de la muestra, dice no contar con las herramientas necesarias para tener sus clases en modalidad virtual, lo que es una gran limitante para su aprendizaje, llegando incluso a la deserción ante la frustración y el desgaste emocional que implica no poder tener los medios necesarios. La tabla 3 muestra las principales Tecnologías de Información y Comunicación-TIC que utilizan los alumnos para realizar sus actividades académicas.

\section{Tabla 3}


TIC empleadas por los alumnos de la FCA-UABJO

\begin{tabular}{ccc}
\hline Tipo de TIC & TIC por alumno & Porcentaje con base al total \\
\hline Computadora & 308 & $53 \%$ \\
Teléfono Celular & 530 & $90 \%$ \\
Tableta & 23 & $4 \%$ \\
Internet & 290 & $49 \%$ \\
Ninguna & 8 & $1 \%$
\end{tabular}

Nota: Es posible determinar el porcentaje de alumnos que utilizan uno o más Tecnologías de Información y Comunicación para acceder a sus clases de manera virtual. El $90 \%$ de los alumnos emplean el celular, seguido de un $53 \%$ que hacen uso de un equipo de computo. Un dato importante es que del total de alumnos el 49\% cuenta con servicio de internet en casa. Información recabada con las encuestas aplicadas a los alumnos de la FCA-UABJO

Un elemento importante para considerar es que, aunque existen casos en que los alumnos cuentan con dos dispositivos, lo que no garantiza un uso adecuado vinculado directamente con su educación. Si podemos observar en la tabla 3, del total de alumnos encuestados que son 583 sólo el $49 \%$ cuenta con servicio de internet en casa, en su mayoría con un $90 \%$ cuentan con teléfono celular, pero ello no es indicio de capacidad del equipo, ni de sus características y compatibilidad con las plataformas educativas empleadas por los profesores. Un dato que hace mucho cuestionar las condiciones en el proceso de aprendizaje es que un 53\% tiene un equipo de computo, pero un $4 \%$ de ese total no cuenta con servicio de internet para poder potencializarlo al máximo.

Del $52 \%$ de los alumnos que respondieron las encuestas manifestaron que el principal obstáculo para poder cumplir con las tareas e investigaciones que sus profesores les pedían realizar fue la falta de cobertura y conectividad a internet. Como se observó anteriormente, debido a la contingencia sanitaria por el COVID-19 un gran número de alumnos tuvo que regresar a sus comunidades, afectando su aprendizaje y obtención del conocimiento planeado al inicio del semestre. El 14\% manifestó que por no contar internet en su comunidad tenían que adquirir otro tipo de planes para tener acceso, lo que les ocasionaba un gasto no contemplado y ello impedía cumplir con los tiempos y las actividades solicitadas por sus maestros. Por otra parte, se encontró que $32 \%$ de los alumnos tuvieron que modificar sus planes y combinar diversas actividades que les complicaba mucho tomar adecuadamente sus clases en esa nueva modalidad.

Una vez determinados los procesos de capacitación correspondiente y puesta en marcha por parte de la Facultad de Contaduría y Administración los recursos tecnológicos para el desarrollo de actividades académicas, se detectó con las encuestas que el $94 \%$ de los profesores utilizaron la plataforma oficial de google para impartir sus clases, mientras que el $6 \%$ restante optó por otras alternativas que consideraron ideales para impartir su clase. Entre las herramientas recurrentes se encontró Google Meet, Zoom y Whats app para videollamadas. En este sentido los alumnos señalaron que en $70 \%$ de los casos las horas de conexión que son similares, la plataforma oficial contratada con 
google se satura y desconecta continuamente a los alumnos y profesores, generando interrupciones en partes importantes de la clase, y aunque se tiene la autorización para grabarlas, esos cortes se hacen presentes en las grabaciones.

Al analizar los resultados relacionados con el apego al horario de clases; el 53\% de los alumnos manifestaron que los catedráticos de la Facultad de Contaduría y Administración si respetaron sus horarios de clase lo que permitió que los alumnos pudieran organizar sus tiempos de clase y el $47 \%$ de los catedráticos no respetaron sus horarios de clases complicándoles tomar sus clases en tiempo y forma, con sus demás materias. En este sentido el $70 \%$ de los alumnos señala no tener el aprendizaje esperado y el $30 \%$ se manifestó satisfecho con las expectativas al inicio del ciclo escolar.

Uno de los principales elementos a identificar con el estudio, tiene relación con los efectos que tuvieron los alumnos en sus clases, determinando específicamente diversas limitaciones y dificultades en el proceso de aprendizaje, mismos que son mostrados en la Tabla 4, y que señalan principalmente incompatibilidad de horarios, falta de recursos tecnológicos, comprensión del tema, nulo aprendizaje, estrés, fala de concentración, espacio adecuado para tomar sus clases derivado de diversos distractores y conexión intermitente.

\section{Tabla 4.}

Limitaciones y dificultades manifiestas por alumnos FCA-UABJO

\begin{tabular}{lcc}
\hline Limitaciones y Dificultades & Alumnos con dificultades (583) & Porcentaje \\
\hline Incompatibilidad de Horarios & 297 & $51 \%$ \\
Falta de recursos tecnológicos & 115 & $20 \%$ \\
Comprensión de temas & 171 & $29 \%$ \\
Nulo aprendizaje & 116 & $20 \%$ \\
Aumento de Estrés & 192 & $33 \%$ \\
Falta de Concentración & 196 & $34 \%$ \\
Falta de Espacio adecuado para clases en línea & 470 & $81 \%$ \\
Distractores varios & 496 & $85 \%$ \\
Conexión intermitente & 49 & $86 \%$ \\
\hline
\end{tabular}

Nota: Uno de los motivos que presenta una mayor incidencia y una limitante fundamental para las clases es la conexión de internet intermitente, donde un $86 \%$ de los alumnos señaló tener ese problema. El $85 \%$ manifestó presentar diversos tipos de distractores. Mientras que el $81 \%$ de los estudiantes señaló no tener el espacio ideal para tomar sus clases. La incompatibilidad en los horarios provocó la ausencia de los estudiantes en un 30\%. Información recabada con las encuestas aplicadas a los alumnos de la FCAUABJO

Las condiciones geográficas en la que se encuentra una gran parte de la población estudiantil muestran un panorama que podría considerarse general, y aunque el abordaje que se realiza en el estudio se centra en el nivel universitario, es posible determinar una generalidad a nivel Universidad. Las 
sobrecargas de trabajo han llevado tanto a los alumnos como a los docentes a implementar estrategias que brinden un mayor aprovechamiento. Sin embargo, las condiciones en el estado no son las propicias para atender plenamente esta problemática.

\section{DISCUSIÓN}

La Organización de las Naciones Unidas (ONU,2020) ha manifestado la brecha existente y la reducción de oportunidades en los sectores vulnerables de la población. Se considera que el $54.2 \%$ de la población oaxaqueña se encuentran en condiciones de pobreza y marginación. Esta situación sin lugar a duda afecta a la población estudiantil que al tener que emigrar a la ciudad de Oaxaca para su preparación universitaria presentan muchas limitaciones y dificultades para su formación.

La CEPAL y UNESCO (2020), hacen hincapié en las afectaciones de las actuales y futuras generaciones en su proceso de formación académica. La realidad demuestra la falta de preparación, previsión y visión gubernamental, institucional y personal para el manejo de la modalidad virtual ante esta situación de pandemia.

Como parte del objetivo, se identificaron las limitaciones y dificultades de los alumnos de la FCA de la UABJO durante la emergencia sanitaria siendo las principales: Incompatibilidad de horarios por parte de los alumnos y docentes para las clases, la falta de recursos tecnológicos ya sea por cuestiones económicas o la orografía de las zonas que no permitian una adecuada conexión a internet, la comprensión de los temas dadas las intermitencias de conexión y la falta de interacción por parte de los profesores. En otros casos se vislumbra un nulo aprendizaje, por la apatía, falta de interés, estrés en aumento, distractores, espacio inadecuado para tomar las clases y como muchos alumnos retornaron a sus zonas geográficas de origen, se vieron en la necesidad de multiplicar sus actividades en el hogar, trabajo y escuela, por lo que la disposición de tiempo para estudiar no era igual que en la modalidad presencial. El supuesto se considera aceptado, ya que los alumnos señalaron que al al emigrar a las clases virtuales no se obtuvo el el mismo conocimiento y su aprendizaje disminuyó.

Dentro de las dificultades se pueden señalar la falta de capacidad y saberes digitales de docentes y alumnos para hacer frente a la modalidad virtual, así como la frustración por no comprender las actividades, la falta de atención. $Y$ compromiso de ambas partes en el proceso enseñanzaaprendizaje. Al ser una investigación exploratoria, se considera no ser concluyente, además que el fenómeno sigue en proceso de análisis; pese a ello el supuesto planteado es aceptado dados los resultados obtenidos con la aplicación de encuestas a los alumnos.

\section{CONCLUSIONES}


Dentro del análisis expuesto en este estudio se aplicaron 583 encuestas a través de google forms, muestra que representa el $17.94 \%$ del total de los estudiantes inscritos en la Facultad de Contaduría y Administración. De los alumnos encuestados el $57 \%$ de los alumnos regresaron a sus comunidades y el $43 \%$ permaneció en la ciudad. Relacionado con la capacidad de la Facultad de Contaduría y Administración para enfrentar el cambio de programa presencial a virtual el $84.39 \%$ de los estudiantes manifestó que no se contaba con la preparación tecnológica ni de los docentes para la transición exitosa a esta nueva modalidad. Por otro lado, el $70.32 \%$ de alumnos manifestaron que un $11 \%$ de los maestros con los cuales toman clases tuvieron la capacidad para adaptar sus programas a una modalidad virtual.

Otro impedimento que tuvieron para no obtener un buen aprendizaje fue, el no contar con las herramientas tecnológicas para sus clases virtuales debido a que en sus comunidades no cuentan con conectividad a internet, lo que les ocasionó un gasto mayor porque tenían que comprar fichas por hora de internet y además no de buena calidad lo que hace imposible tener una buena cobertura. Tomando en cuenta que el $93.48 \%$ de los catedráticos si utilizaron alguna plataforma para cumplir sus temarios y contenidos.

El $69.81 \%$ de los alumnos encuestados manifestaron que, por estas variables, no obtuvieron el aprendizaje o conocimiento esperado: falta de comprensión, falta de concentración, dudas no aclaradas, adaptarse a los horarios de los catedráticos, por lo que el $94.34 \%$ manifestó de ser posible cuando se regrese al semestre 2020-2 se pueda hacer o retomar cursos remediales o de retroalimentación.

Esta nueva realidad, ha mermado en los jóvenes un entorno de enriquecimiento y estímulo que les permita crear oportunidades de aprendizaje e interacción social presente en la educación presencial. Si sumamos el hecho que existen zonas en las que el acceso de internet es nulo o bien la conexión tiene muy malas condiciones; sumado a que existen alumnos que carecen de equipo de cómputo y tienen que gastar más para poder rentar una computadora o pagar datos, se abre una brecha digital y de aprendizaje más grande entre quienes pueden tener acceso y quienes tienen que buscar alternativas más caras o desertar de sus estudios. Por lo tanto, uno de los elementos importantes a considerar ante esta contingencia es la innovación educativa y tecnológica para hacer frente a las pérdidas en materia de aprendizaje y prevenir el abandono escolar, especialmente en aquellos alumnos que viven en condiciones de pobreza y marginación.

La preparación del docente debe incorporar además un apoyo para tener una conectividad adecuada y dar seguimiento al aprendizaje y generar la flexibilidad entre lo académico y lo profesional. Las prioridades a las que deben enfocarse las Instituciones de Educación Superior son: recuperar las pérdidas en los procesos de aprendizaje; monitorear los casos de estudiantes en riesgo de abandono y 
brindar los apoyos necesarios para evitar la deserción; generar los mecanismos necesarios para centrarse en el bienestar social y emocional de docentes, personal administrativo y alumnos a través de programas institucionales y con apoyos de instancias gubernamentales. Estos elementos deberán permitir prevenir una crisis en materia de aprendizaje que conlleve a una catástrofe generacional que sin lugar a duda afectará los entornos de trabajo, y frenarán el progreso económico y el desarrollo sostenible.

\section{REFERENCIAS}

Consejo Nacional de Evaluación de la Política de Desarrollo Social [CONEVAL] (2015). Medición de la pobreza multidimensional, Indicadores a nivel municipal. Obtenido de https://www.coneval.org.mx/InformesPublicaciones/FolletosInstitucionales/Documents/Medicionmultidimensional-de-la-pobreza-en-Mexico.pdf

Comisión Económica para América Latina y el Caribe [CEPAL] (Abril de 2020). América Latina y el Caribe ante la pandemia del COVID-19: efectos económicos y sociales. Santiago: CEPAL. Obtenido de Informe Especial COVID-19, № 1. Obtenido de https://www.cepal.org/es/publicaciones/45337america-latina-caribe-la-pandemia-covid-19-efectos-economicos-sociales

Comisión Económica para América Latina y el Caribe [CEPAL], Organización de las Naciones Unidas para la Educación, la Ciencia y la Cultura [UNESCO] (Agosto de 2020). La educación en tiempos de la pandemia de COVID-19. Obtenido de https://repositorio.cepal.org/bitstream/handle/11362/45904/1/S2000510_es.pdf

Comisión Económica para America Latina y el Caribel [CEPAL] (Mayo de 2020). Informe sobre el impacto económico en América Latina y el Caribe de la enfermedad por coronavirus (COVID-19).

Obtenido de https://www.cepal.org/es/publicaciones/45602-informe-impacto-economico-americalatina-caribe-la-enfermedad-coronavirus-covid

Banco Mundial (13 de Abril de 2020). Posibles escenarios de pérdida en los aprendizajes durante el cierre de escuelas. Obtenido https://blogs.worldbank.org/es/education/se-debe-evitar-aplanar-lacurva-de-la-educacion-posibles-escenarios-de-perdida-en-los

Gobierno del Estado de Oaxaca (2020). Plan Estatal de Desarrollo 2016-2022. Obtenido de https://www.finanzasoaxaca.gob.mx/pdf/planes/Plan_Estatal_de_Desarrollo_2016-2022.pdf

Haeck, C., y Lefebvre, P. (03-20 de Junio de 2020). Pandemic school closures may increase inequality in test scores. DOI: 10.3138/cpp.2020-055 IESALC. (2020). UNESCO. Obtenido de COVID-19 y educación superior: De los efectos inmediatos al día después. Análisis de impactos, respuestas políticas y recomendaciones: http://www.iesalc.unesco.org/wp-content/uploads/2020/05/COVID19-ES-130520.pdf

Instituto Internacional para la Educación Superior en America Latina y el Caribe [IESALC] (2020). COVID19 y educación superior: De los efectos inmediatos al día después. Análisis de impactos, 
respuestas políticas y recomendaciones. UNESCO.Obtenido de http://www.iesalc.unesco.org/wpcontent/uploads/2020/05/COVID-19-ES-130520.pdf

Oganización de las Naciones Unidas [ONU]. (Agosto de 2020). La educación durante la COVID-19 y despues de ella. Organización de las Naciones Unidas. Obtenido de https://www.un.org/sites/un2.un.org/files/policy_brief_-_education_during_covid19_and_beyond_spanish.pdf

Organización Panamericana de la Salud. (2020). Coronavirus. Obtenido de https://paho.org/es/temas/coronavirus

Universidad Autónoma Benito Juárez de Oaxaca [UABJO](Marzo-Mayo de 2020 a). Cronología de las acciones realizadas por la Universidad Autónoma "Benito Juárez" de Oaxaca, para dar continuidad a las actividades esenciales ante la contingencia sanitaria. Obtenido de Covid19.uabjo.mx:

http://www.covid19.uabjo.mx/media/42/2020/06/CronologiaAccionesUABJOCOVID19.pdf Universidad Autónoma Benito Juárez de Oaxaca [UABJO] (18 de Agosto de 2020 b). La FCA ofrecerá servicios académicos de manera virtual. Obtenido de uabjo.mx: http://www.uabjo.mx/la-fcaofrecera-servicios-academicos-de-maneravirtual\#: :text=\%2D\%20Ante\%20la\%20situaci\%C3\%B3n\%20que\%20prevalece,2020\%2D2021\% 2C\%20en\%201\%C3\%ADnea. 